\title{
ChemComm
}

Cite this: Chem. Commun., 2013, 49, 4896

Received 23rd February 2013, Accepted 6th April 2013

DOI: $10.1039 / c 3 c c 41411 f$

\section{Metal carbonyls supported on iron oxide nanoparticles to trigger the CO-gasotransmitter release by magnetic heating $\dagger$}

\author{
Peter C. Kunz, ${ }^{* a}$ Hajo Meyer, ${ }^{a}$ Juri Barthel, ${ }^{b}$ Silvio Sollazzo, ${ }^{c}$ Annette M. Schmidt* \\ and Christoph Janiak*a
}

www.rsc.org/chemcomm

\begin{abstract}
Magnetic iron oxide, maghemite $\left(\mathrm{Fe}_{2} \mathrm{O}_{3}\right)$ nanoparticles with covalent surface-bound CO-releasing molecules (CORMs) can be triggered to release $\mathrm{CO}$ through heating in an alternating magnetic field. In the proof-of-concept study the rate of CO-release from $\left[\mathrm{RuCl}\left(\mathrm{CO}_{3}\right)\right.$ ( $\mu$-DOPA)]@maghemite nanoparticles was doubled upon exposure to an external alternating magnetic field $\left(31.7 \mathrm{kAm}^{-1}, 247 \mathrm{kHz}\right.$, $25{ }^{\circ} \mathrm{C}, 39.9$ mTesla, DOPA = dioxyphenyl-alaninato).
\end{abstract}

Small gaseous molecules, so-called gasotransmitters, represent important signal molecules in biological systems. Much research has already been devoted to the multiple biological functions of nitrogen monoxide $(\mathrm{NO}),{ }^{1}$ so the signal-transfer properties of carbon monoxide $(\mathrm{CO})$ and hydrogen sulfide $\left(\mathrm{H}_{2} \mathrm{~S}\right)$ are now gaining attention. ${ }^{2-4}$ The discovery of the cell regulating function of $\mathrm{CO}$ was prevented for a long time by its known toxicity in medium and higher doses. Carbon monoxide shows cell protective, antiinflammatory and vasodilatatory (blood vessel relaxing) effects which confer it with great potential as a therapeutic agent. ${ }^{3}$ It is difficult to correctly dose gaseous CO. Further, CO has a high affinity to hemoglobin and cytochromes. This has prevented, so far, clinical usage of CO despite its promising properties. The binding of CO to hemoglobin and cytochromes endangers the oxygen supply upon overdosage and is also responsible for its low bioavailability. ${ }^{4}$

The controlled dose-release of $\mathrm{CO}$ at the desired target tissue is a major prerequisite for a meaningful therapeutic usage. The utilization of CO-releasing molecules (CORMs, Scheme 1) is a promising possibility for the release of defined amounts of $\mathrm{CO}$ at the target tissue. ${ }^{5}$ The water soluble Ru-complex CORM-3 (Scheme 1) releases CO under physiological conditions and shows vasodilatatoric, anti-inflammatory, renal protective, anti-ischemic

\footnotetext{
${ }^{a}$ Institut für Anorganische Chemie und Strukturchemie, Universität Düsseldorf, 40204 Düsseldorf, Germany.E-mail: peter.kunz@uni-duesseldorf.de, janiak@uni-duesseldorf.de

${ }^{b}$ Ernst Ruska-Centrum, Forschungszentrum Jülich, D-52425 Jülich, Germany ${ }^{c}$ Institut für Physikalische Chemie, Universität zu Köln, Luxemburger Str. 116, D-50939 Köln, Germany. E-mail: annette.schmidt@uni-koeln.de

$\dagger$ Electronic supplementary information (ESI) available: Detailed syntheses and myoglobin assay procedures, also for $\mathbf{4}$; nanoparticles size determination by DLS and TEM, magnetic properties. See DOI: 10.1039/c3cc41411f
}

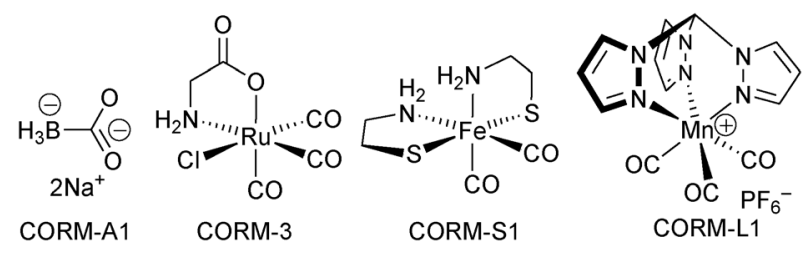

Scheme 1 Examples of CO-releasing molecules (CORMs) for biological investigations.

and anti-apoptotic effects in pre-clinical studies. ${ }^{6}$ The CO-release is induced by a hydrolytic mechanism with pseudo-first order kinetics and a half-life in the minute range. ${ }^{7}$ The physiologically undefined and fast release limits the usage of CORM-3 considerably. An important progress was the development of CORMs which can be activated by an external stimulus (trigger) to release CO. An example of such activable CORMs is the boranate carbonate CORM-A1 which exhibits a $\mathrm{pH}$-dependent CO-release. ${ }^{8}$ Also, the photoinduced decarbonylation ${ }^{9}$ and enzymatic activation ${ }^{10}$ of transition-metal complexes are mechanisms for activable systems.

Yet, the problem of spatially restricted release in the target tissue still remains a challenge. A possible solution to this problem is the use of nanoparticle carriers which allow both a controlled guidance to the desired position as well as the time and spatially controlled release of CO under local fields. Previous work has addressed the use of nanoparticular carriers for CORMs with spontaneous or photoinduced CO-release. ${ }^{11}$ Here, we present the use of biocompatible magnetic iron oxide nanoparticles (IONPs) as carriers for CORMs (CORM@IONP). In a proof-of-concept study we demonstrate the triggered CO-release through induced heating in an alternating magnetic field (Scheme 2).

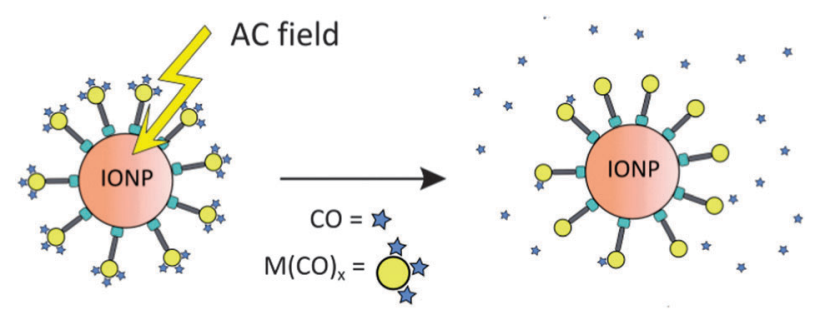

Scheme 2 Schematic presentation of induced CO-release from CORM-functionalized iron oxide nanoparticles (IONP) through an alternating magnetic field. 
The heating of magnetic nanoparticles in alternating magnetic fields due to loss mechanisms (hyperthermia) has been investigated for some years now for the thermo-ablation of diseased tissue in cancer therapy. ${ }^{12}$ A macroscopic temperature increase is possible with sufficient particle concentration in the affected tissue. Furthermore, magnetic nanoparticles allow for concentration in physiological environments by magnetic control $^{13}$ and can be used as transporters for the release of drug compounds. ${ }^{14}$ Theoretical calculations suggest that a thermal stationary state is quickly reached upon electromagnetic excitation of nanoscopic (metallic or magnetic) particles due to their high surface-to-volume-ratio. It is agreed that there is a negligible temperature difference between the bulk phase of the particle and its surrounding area. Depending on the particle size and the surrounding medium an initiation time of about 100 ps can be assumed. ${ }^{15}$ Experiments indicate that the energy transfer between a particle and the surrounding phase leads to increased excitation of vibrational degrees of freedom in the surface-bound molecules. This can be used for the activation of release processes, ${ }^{14 a, d}$ local phase transitions, ${ }^{14 a, b}$ targeted protein denaturation or activation $^{14 c}$ and oligonucleotide dehybridization. ${ }^{16}$

To test the concept of magnetic-nanoparticle heating for the induced CO-release from supported CORMs, a suitable CORMcompound was immobilized on the surface of maghemite $\left(\mathrm{Fe}_{2} \mathrm{O}_{3}\right)$ nanoparticles. First, the iron oxide particle surface was functionalized with $\mathrm{D} / \mathrm{L}$-3-(3,4-dihydroxyphenyl)alanine (D/L-DOPA) to provide an anchor group for the subsequent introduction of the CORM-fragment. Product 2 (Scheme 3) was collected using a magnet, washed and reacted in methanol with $\left[\mathrm{Ru}_{2} \mathrm{Cl}_{4}(\mathrm{CO})_{6}\right]$ to yield CORM-functionalized nanoparticles 3 (Scheme 3). CORM@IONP, 3, contains a derivative of CORM-3 where the ruthenium carbonyl fragment carries a dioxyphenylalaninato instead of a glycinato ligand. The former is applicable in IONP surface immobilization through its dioxyphenyl or catecholate unit. We intentionally chose a system which allows for comparison with the well-investigated, fast CO-releaser CORM-3 in order to have short kinetic measurements in the investigation of this novel release concept. For additional control experiments the molecular complex tri(carbonyl)-chloridophenylalaninato-ruthenium(II) 4 was used.

Functionalization of the maghemite nanoparticles can be followed using IR-spectroscopy (Fig. S3 in ESI $\dagger$ ). The strong CO

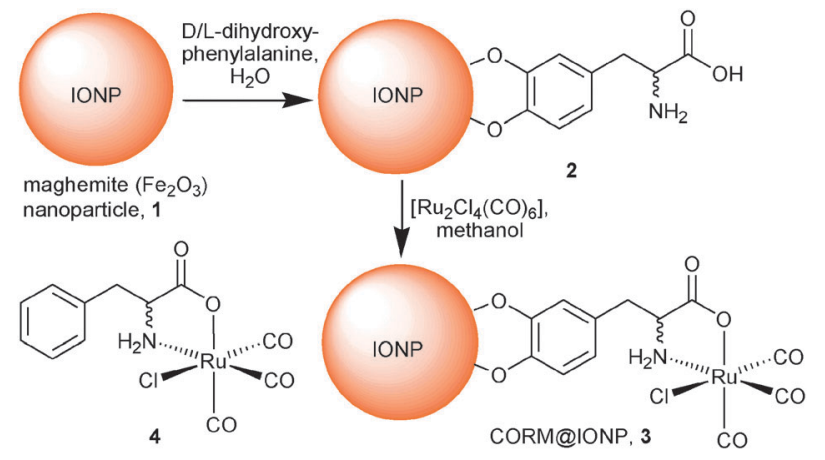

Scheme 3 Synthesis of CORM-functionalized nanoparticles 3 and the tri(carbonyl)-chlorido-phenylalaninato-ruthenium(॥) model compound 4.

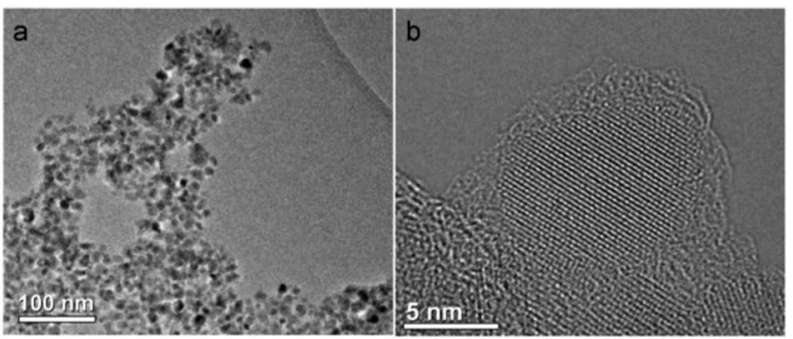

Fig. 1 TEM-images of CORM@IONP, 3. (a) Agglomerated (magnetic) nanoparticles, (b) a high-resolution image of one representative particle with lattice planes and a ligand shell; further images are provided in the ESI. $†$

stretching vibrations of the $\mathrm{Ru}(\mathrm{CO})_{3}$-fragment in 3 were observed at around 2059 and $1986 \mathrm{~cm}^{-1}$, which agree with the vibrational frequencies of the analogous CORM-3 under basic conditions (see also detailed IR description in ESI $\dagger$ ). ${ }^{17}$

The maghemite NPs 1 have a hydrodynamic diameter of $(8 \pm 2) \mathrm{nm}$ which increases to $(11 \pm 3) \mathrm{nm}$ for 2 after functionalization with $\mathrm{D} / \mathrm{L}$-dihydroxyphenylalanine (both dynamic light scattering, DLS, see ESI $\dagger$ ). This value does not change through reaction with $\left[\mathrm{Ru}_{2} \mathrm{Cl}_{4}(\mathrm{CO})_{6}\right]$ to give 3 . The ligand-/CORM-shell of 3 can be clearly seen in the high-resolution transmission electron micrograph (TEM). Fig. 1b shows a representative nanoparticle of 3 with its maghemite lattice planes along the crystallographic [222] direction and the ligand corona. The maghemite core diameter in 3 is $(9 \pm 2) \mathrm{nm}$ as shown by TEM and the visible additional organic shell has a width of around $2 \mathrm{~nm}$ which agrees very well with the value derived from DLS for 2.

A Ru: Fe atom ratio of $1: 25$ was determined using ICP-MS, that is, for each nanoparticle, there are about $400 \mathrm{Ru}$ complexes on the surface, corresponding to less than $60 \%$ of the estimated possible coverage (see ESI $\dagger$ ). The magnetic properties of nanoparticles 2 were determined using vibrating sample magnetometry (VSM). Lack of hysteresis shows superparamagnetic behavior of the nanoparticles both in dispersion as well as in the solid state. The saturation magnetization yields a high mass fraction of the magnetic phase of about 67\% (see Table S2, $\mathrm{ESI} \dagger$ ) from which a volume-averaged core diameter of $12 \mathrm{~nm}$ is calculated (see ESI, $\uparrow$ Fig. S7, Table S2).

The CO-release was followed with a myoglobin assay using UV/Vis-spectroscopy (see ESI $\dagger$ ). ${ }^{18,19}$ For CORM@IONP 3 a singlestep pseudo-first order CO-release can be deduced without a magnetic field from the relative decrease and increase in the absorption at 541, 556 and 578 nm (Fig. 2 with Fig. S8 and S9, ESI $\dagger$ ). This observation is analogous to those for 4 (Fig. S12 in ESI $\dagger$ ) and CORM-3. Yet, the half-life for the CO-release from 3 with $t_{\frac{1}{2}}=(13 \pm 2) \min \left(25^{\circ} \mathrm{C}\right)$ is much longer than those from 4 $\left[t_{\frac{1}{2}}=(2.7 \pm 0.2) \mathrm{min}, 20{ }^{\circ} \mathrm{C}\right]$ and CORM-3 $\left(t_{\frac{1}{2}}=2.3 \mathrm{~min}, 30{ }^{\circ} \mathrm{C}\right) .{ }^{19}$ This surface stabilization of the CORM towards solvolytic and protein-reaction induced ${ }^{20}$ CO-release is explained by a diffusion barrier in the surface layer and by steric hindrance from the surrounding CORM molecules and free D/L-DOPA ligands. A similar effect was described for a CORM in micelles. ${ }^{11 c}$

Application of an external alternating magnetic field to CORM@IONP 3 (31.7 kAm ${ }^{-1}, 247 \mathrm{kHz}, 25{ }^{\circ} \mathrm{C}, 39.9$ mTesla) 
(a)
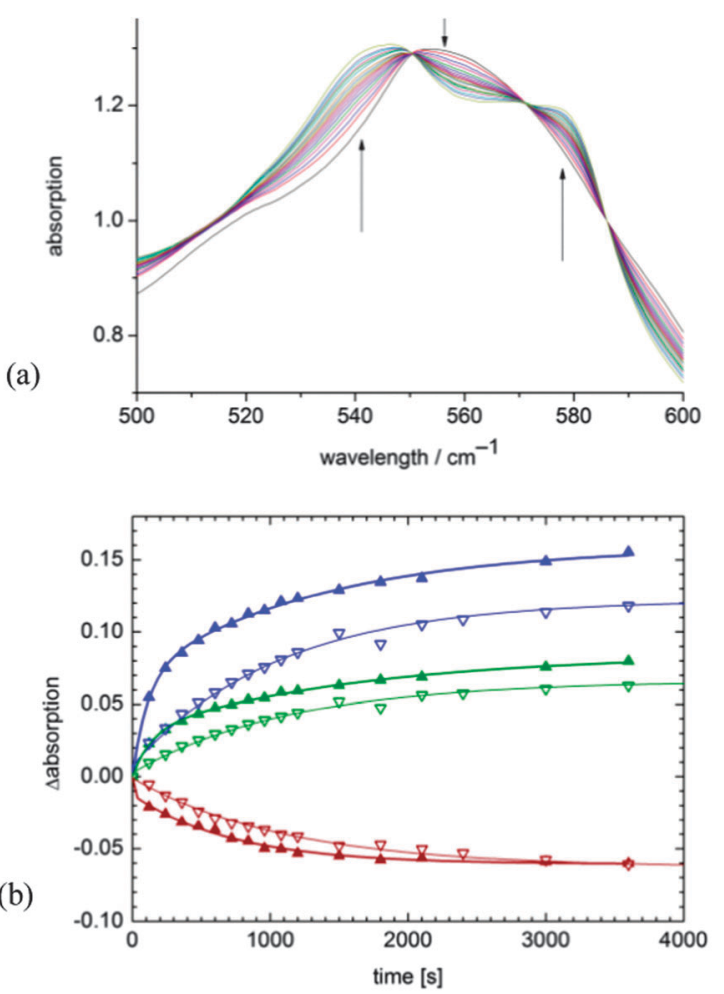

Fig. 2 (a) Typical change in the absorption bands of reduced skeletal muscle horse myoglobin $(76 \mu \mathrm{M})$ between $500-600 \mathrm{~nm}$ during the CO-release from CORM@IONP 3, here with an alternating magnetic field $\left(247 \mathrm{kHz}, 31.7 \mathrm{kAm}^{-1}\right.$, 39.9 mTesla) at $25^{\circ} \mathrm{C}$. Intensity changes were followed at 541,556 and $578 \mathrm{~nm}$ to give the typical CO-release curves in (b) from which the decrease in half-life with a magnetic field can be quantified. Empty symbols $(\nabla)$, without a field; filled symbols $(\boldsymbol{\Lambda})$, with an alternating magnetic field; blue symbols $(\nabla, \boldsymbol{\Lambda})$, change in the intensity at $541 \mathrm{~nm}$; red-brown symbols $(\nabla, \boldsymbol{\Lambda})$, change in the intensity at $556 \mathrm{~nm}$; green symbols $(\nabla, \Delta)$, change in the intensity at $578 \mathrm{~nm}$

yields a two-fold accelerated CO-release rate with a half-life of $t_{\frac{1}{2}}=(7 \pm 2)$ min (Fig. 2, Table S3 in ESI $\dagger$ ). It is important to note that the temperature in the dispersion did not increase in the thermostated cuvette during the application of the magnetic field. Also, some deviation from a simple (pseudo-) first order kinetic can be seen for the CO-release (Fig. S9 in ESI†). Future studies shall elucidate this behavior. An enhanced CO-release through excitation of the $\mathrm{Ru}(\mathrm{CO})_{3}$-fragment induced by an alternating field can be excluded. The half-life values for CO release from the phenylalaninato-model compound $\mathbf{4}$ without and with an alternating magnetic field (no IONP present) were identical $[(2.7 \pm 0.2) \mathrm{min}$ and $(3.0 \pm 0.4) \mathrm{min}]$ within experimental error.

The observed rate increase shows the principal possibility for a triggered (induced) CO-release in target tissue with the nanoparticle nuclei acting as local field antennae. The accelerated CO-release clearly shows the field-induced surface effect (Scheme 2, Fig. 2 and Fig. S10, ESI $\dagger$ ) despite the fast heat dissipation and a low particle concentration which prevents a macroscopic heating effect in the dispersion.

CORM-functionalized iron oxide nanoparticles have yielded a new possibility of inducing a faster CO-release through an external alternating magnetic field. Furthermore this concept which is introduced here opens new ways to enrich tissue-specific CORMs, by magnetic focusing or through attachment of additional tissueor cell-addressing ligands on the IONP surface.

The work of CJ was supported by DFG grant Ja466/17-1.

\section{Notes and references}

1 D. A. Riccio and M. H. Schoenfisch, Chem. Soc. Rev., 2012, 41, 3731-3741; A. W. Carpenter and M. H. Schoenfisch, Chem. Soc. Rev., 2012, 41, 3742-3752; P. N. Coneski and M. H. Schoenfisch, Chem. Soc. Rev., 2012, 41, 3753-3758.

2 A. K. Mustafa, M. M. Gadalla and S. H. Snyder, Sci. Signaling, 2009, 2, re2.

3 S. W. Ryter and L. E. Otterbein, BioEssays, 2004, 26, 270-280; B. E. Mann, Organometallics, 2012, 31, 5728-5735; R. Motterlini, B. Haas and R. Foresti, Med. Gas Res., 2012, 2, 28.

4 B. E. Mann, Topics in Organometallic Chemistry, Springer, Berlin, Heidelberg, 2010.

5 C. C. Romão, W. A. Blättler, J. D. Seixas and G. J. L. Bernardes, Chem. Soc. Rev., 2012, 41, 3571-3583; T. Johnson, B. E. Mann, J. Clark, R. Foresti and C. Green, Angew. Chem., Int. Ed., 2003, 42, 3722-3729.

6 R. Motterlini and L. E. Otterbein, Nat. Rev. Drug Discovery, 2010, 9, 728-743; R. Alberto and R. Motterlini, Dalton Trans., 2007, 1651-1660; R. Motterlini, B. E. Mann, T. Johnson, J. Clark, R. Foresti and C. Green, Curr. Pharm. Des., 2003, 9, 2525-2539.

7 R. Motterlini, B. E. Mann and R. Foresti, Expert Opin. Invest. Drugs, 2005, 14, 1305-1318.

8 R. Motterlini, P. Sawle, S. Bains, J. Hammad, R. Alberto, R. Foresti and C. Green, FASEB J., 2005, 18, 284-286.

9 A. E. Pierri, A. Pallaoro, G. Wu and P. C. Ford, J. Am. Chem. Soc., 2012, 134, 18197-18200; W. Huber, R. Linder, J. Niesel, U. Schatzschneider, B. Spingler and P. C. Kunz, Eur. J. Inorg. Chem., 2012, 3140; V. P. L. Velásquez, T. M. A. Jazzazi, A. Malassa, H. Gorls, G. Gessner, S. H. Heinemann and M. Westerhausen, Eur. J. Inorg. Chem., 2012, 1072-1078; D. Crespy, K. Landfester, U. S. Schubert and A. Schiller, Chem. Commun., 2010, 46, 6651-6662; R. D. Rimmer, H. Richter and P. C. Ford, Inorg. Chem., 2010, 49, 1180-1185; U. Schatzschneider, Eur. J. Inorg. Chem., 2010, 1451-1467; P. C. Kunz, W. Huber, A. Rojas, U. Schatzschneider and B. Spingler, Eur. J. Inorg. Chem., 2009, 5358-5366.

10 S. Romanski, B. Kraus, M. Guttentag, W. Schlundt, H. Rücker, A. Adler, J.-M. Neudoerfl, R. Alberto, S. Amslinger and H. G. Schmalz, Dalton Trans., 2012, 41, 13862-13875.

11 (a) N. E. Brückmann, M. Wahl, G. J. Reiß, M. Kohns, W. Wätjen and P. C. Kunz, Eur. J. Inorg. Chem., 2011, 4571-4577; (b) G. Dördelmann, H. Pfeiffer, A. Birkner and U. Schatzschneider, Inorg. Chem., 2011, 50, 4362-4367; (c) U. Hasegawa, A. J. van der Vlies, E. Simeoni, C. Wandrey and J. A. Hubbell, J. Am. Chem. Soc., 2010, 132, 18273-18280.

12 A. J. Cole, V. C. Yang and A. E. David, Trends Biotechnol., 2011, 29, 323-332.

13 A. S. Lübbe, C. Alexiou and C. Bergemann, J. Surg. Res., 2001, 95, 200-206; C. Alexiou, W. Arnold, R. J. Klein, F. G. Parak, P. Hulin, C. Bergemann, W. Erhardt, S. Wagenpfeil and A. S. Lübbe, Cancer Res., 2000, 60, 6641-6648.

14 (a) A. M. Schmidt, J. Magn. Magn. Mater., 2005, 289, 5-8; (b) R. Messing and A. M. Schmidt, Prog. Colloid Polym. Sci., 2008, 134, 134-140; (c) T. Gelbrich, G. U. Marten and A. M. Schmidt, Polymer, 2010, 51, 2818-2824; (d) G. U. Marten, T. Gelbrich and A. M. Schmidt, Beilstein J. Org. Chem., 2010, 6, 922-931.

15 M. Perner, P. Bost, U. Lemmer, G. von Plessen, J. Feldmann, U. Becker, M. Mennig, M. Schmitt and H. Schmidt, Phys. Rev. Lett., 1997, 78, 2192; P. Keblinski, D. Cahill, A. R. Bodapati and T. A. Tato, J. Appl. Phys., 2006, 100, 1-5; I. Levine, O. Shenkman, M. Winkler, A. M. Schmidt and M. Gottlieb, Macromol. Symp., 2010, 291-292(1), 278-286.

16 J. Stehr, C. Hrelescu, R. Sperling, G. Raschke, M. Wunderlich, A. Nichtl, D. Heindl, K. Kürzinger, W. J. Parak, T. A. Klar and J. Feldmann, Nano Lett., 2008, 8, 619-623; M. Reismann, J. C. Bretschneider, G. von Plessen and U. Simon, Small, 2008, 4, 607-610.

17 T. R. Johnson, B. E. Mann, I. P. Teasdale, H. Adams, R. Foresti, C. J. Green and R. Motterlini, Dalton Trans., 2007, 1500-1508.

18 A. J. Atkin, J. M. Lynam, B. E. Moulton, P. Sawle, R. Motterlini, N. M. Boyle, M. T. Pryce and I. J. S. Fairlamb, Dalton Trans., 2011, 40, 5755-5761.

19 S. McLean, B. E. Mann and R. K. Poole, Anal. Biochem., 2012, 427, 36-40.

20 T. Santos-Silva, A. Mukhopadhyay, J. D. Seixas, G. J. L. Bernardes, C. C. Romão and M. J. Romão, J. Am. Chem. Soc., 2011, 133, 1192-1195. 